\title{
Detecting Convoys in Networks of Short-Ranged Sensors
}

\author{
Sean Lawlor and Michael Rabbat \\ Department of Electrical and Computer Engineering, McGill University \\ Montreal, Quebec, Canada \\ Email: slawlor@slawlor.com and michael.rabbat@mcgill.ca
}

\begin{abstract}
Detecting groups of vehicles travelling together as a convoy is an important problem in military and law enforcement applications. License plate recognition sensors provide discrete, irregularly sampled, time series information about where vehicles are travelling. With this irregular time series, we would like to determine when vehicles travel as a convoy.

We construct a semi-Markov process to model network traffic and utilize the Markov property to develop a sequential hypothesis test. This requires defining two models for how vehicles travel through the network and testing the likelihood between them. The main contribution of this work is the modeling of the alternate hypothesis of when two vehicles are traveling as a convoy.

We present performance results based on simulated data showing the tradeoff between false-positives and true detections.
\end{abstract}

Keywords-Convoy Detection, License Plate Recognition, LPR

\section{INTRODUCTION}

Detecting groups of vehicles traveling together as a convoy is an emerging problem in law enforcement. License plate recognition (LPR) cameras are a new technology which can facilitate this task. License plate recognition cameras are static short-ranged sensors which can detect when a vehicle passes in front of them and record the license plate, observation time, latitude and longitude of the observation. Together this information forms an irregularly sampled time-series of observations of vehicles.

This paper devises a method for translating the locations of these sensors into the states of a semi-Markov process which one can then observe the vehicles as traveling through. We then propose a new model for defining how vehicles transition through this semi-Markov process dependent on one another, in other words in a convoy. Finally we devise a statistical hypothesis testing method [2] for determining if the pair of vehicles being tracked are traveling as a convoy versus traveling independent of each other.

\section{A. Related Work}

The problem of tracking groups or pairs of vehicles together as a convoy has received limited attention. The majority of the existing literature on convoy detection/tracking use longrange sensors such as Ground Moving Target Indicator (GMTI) sensors [3], [4]. However this type of sensor provides regularly sampled, continuous information about the vehicles that are being tracked. This makes these methods not applicable for two reasons in this context. The first is that using GMTI data is not suitable for urban environments where long line-of-sight is not available and would result in an overly expensive solution. Secondly the methods used with GMTI tracking and detection are not directly transferable to LPR sensors with the irregular time series samples.

\section{Convoy Detection via Sequential Hypothesis TESTING}

Given a set of short-ranged sensors, this paper defines a method to model vehicles' movements through the network and then detect which of the vehicles are moving following similar paths, therefore defining a convoy.

\section{A. Problem Formulation}

Assume there are $C$ static short-ranged sensors with known locations placed around a network of links which vehicles can travel around. These sensors have no regular sampling rate, they simply record when an vehicle passes by it.

As vehicles move throughout this network, they can only be observed at one of these $C$ sensors. Therefore the only information available are the discrete samples of vehicles when they pass by a sensor. These samples include the time at which the observation occurred, the location of the observation (i.e. the capturing sensor id), and label of the vehicle which was observed.

We would like to detect when pairs of vehicles traveling through this network appear more correlated versus acting independently.

When one tries to analyze this data, it appears as though the vehicles are moving between sensors after some delay. There is no knowledge of the path from sensor $x$ to sensors $y$, just that the vehicle is now at sensor $y$ after being at $x$ after some time. This looks very similar to a first-order Markov model where the vehicles are transitioning from state to state in a state space $S=\{1 \ldots C\}$.

Now consider two vehicles, labeled as $X$ and $Y$, moving through this state space, following unknown routes. Each vehicle can be visualized following a sample route in Figure 


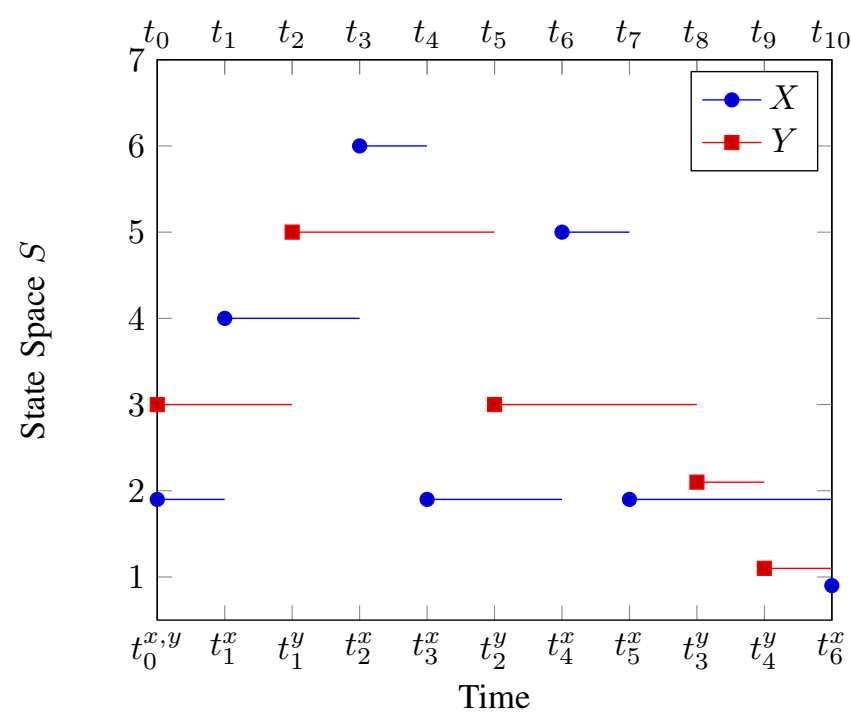

Fig. 1. An example sample path of vehicles $X$ and $Y$ through the state space, $S$.

1 and result in the following sample path observations

$$
\begin{aligned}
& X(t)=\left\{X\left(t_{0}^{x}\right)=x_{0}, X\left(t_{1}^{x}\right)=x_{1}, \ldots, X\left(t_{n_{x}(t)}^{x}\right)=x_{n_{x}(t)}\right\} \\
& =\left\{\left(x_{k}, t_{k}^{x}\right)\right\}_{k=0}^{n_{x}(t)} \\
& \text { and } \\
& Y(t)=\left\{Y\left(t_{0}^{y}\right)=y_{0}, Y\left(t_{1}^{y}\right)=y_{1}, \ldots, Y\left(t_{n_{y}(t)}^{y}\right)=y_{n_{y}(t)}\right\} \\
& =\left\{\left(y_{k}, t_{k}^{y}\right)\right\}_{k=0}^{n_{y}(t)}
\end{aligned}
$$

where $t_{k}^{x}$ is the time of the $k^{t h}$ sample of $X$ and similarly for $Y$. Also $n_{x}(t)$ and $n_{y}(t)$ are defined as

$$
\begin{aligned}
& n_{x}(t)=\max \left\{k: t_{k}^{x} \leq t\right\} \\
& n_{y}(t)=\max \left\{k: t_{k}^{y} \leq t\right\}
\end{aligned}
$$

or equivalently the number of samples of $X$ and $Y$ up to time $t$. The inter-arrival times for vehicles $X$ and $Y$ are represented by $\tau_{i}^{x}=\left(t_{i}^{x}-t_{i-1}^{x}\right)$ and $\tau_{i}^{y}=\left(t_{i}^{y}-t_{i-1}^{y}\right)$, respectively.

\section{B. Markov Model for a Single vehicle}

Before we can define any model which describes how the pair of vehicles travels together through the network, we first define the standard model for how they travel as a single, independent vehicle. In order to better model this process, we need to be able to model the vehicle at any time $t$, not just at the observation time. At time $t$ there are $n_{x}(t)$ observations of $X$. The elapsed time since the last observation is $\tau_{n_{x}(t)}^{x}:=t-t_{n_{x}(t)}$.

The likelihood of the observations $X(t)$ of a single vehicle is

$$
\begin{aligned}
& p_{t}(X(t)) \\
& =\pi\left(x_{0}\right)\left[\prod_{k=1}^{n_{x}(t)} \operatorname{Pr}\left(x_{k} \mid x_{k-1}\right) f_{t}\left(t_{k}^{x} \mid t_{k-1}^{x}, x_{k}, x_{k-1}\right)\right] \\
& \quad \times \operatorname{Pr}\left(t_{k+1}^{x}>t \mid t_{k}^{x}, x_{k}\right) \\
& =\pi\left(x_{0}\right)\left[\prod_{k=1}^{n_{x}(t)} \operatorname{Pr}\left(x_{k} \mid x_{k-1}\right) f_{\tau}\left(t_{k}^{x}-t_{k-1}^{x} \mid x_{k}, x_{k-1}\right)\right] \\
& \quad \times\left(1-F_{\tau}\left(t-t_{k}^{x} \mid x_{k}\right)\right),
\end{aligned}
$$

which is the Markov path probability of $X$ following its observed path at the transition intervals described by $f_{\tau}$ multiplied by the $\mathrm{CDF}$ of the interval waiting time for state $x_{k}$. The CDF in this equation is the probability that $X$ is still waiting at state $x_{k}$ at time $t$. This can alternately be expressed as

$$
p_{t}(X(t))=p_{t}\left(\left\{x_{t}\right\}_{k=0}^{n_{x}(t)}\right) \cdot p_{t}\left(\left\{t_{k}^{x}\right\}_{k=0}^{n_{x}(t)} \mid\left\{x_{k}\right\}_{k=0}^{n_{x}(t)}\right)
$$

where

$$
p_{t}\left(\left\{x_{t}\right\}_{k=0}^{n_{x}(t)}\right)=\pi\left(x_{0}\right) \prod_{k=1}^{n_{x}(t)} \operatorname{Pr}\left(x_{k} \mid x_{k-1}\right)
$$

and

$$
\begin{aligned}
& p_{t}\left(\left\{t_{k}^{x}\right\}_{k=0}^{n_{x}(t)} \mid\left\{x_{k}\right\}_{k=0}^{n_{x}(t)}\right) \\
& =\left(1-F_{\tau}\left(t-t_{n_{x}(t)}^{x} \mid x_{n_{x}(t)}\right)\right) \times \prod_{k=1}^{n_{x}(t)} f_{\tau}\left(t-t_{k} \mid x_{k}, x_{k-1}\right) .
\end{aligned}
$$

\section{Markov Model for Two Independent vehicles}

Now that there is a defined model for a single vehicle traveling through the Markov chain at any time, we can extend this to two vehicles moving independently of each other. This will form the distribution which describes the null $\left(H_{0}\right)$ hypothesis in the hypothesis test defined later. The likelihood of the observed path for both $X$ and $Y$ under $H_{0}$ is

$$
p_{t}\left(X(t), Y(t) \mid H_{0}\right)=p_{t}(X(t)) p_{t}(Y(t))
$$

which is simply the product of the two independent likelihoods defined in Equation 4 Utilizing this definition for the null hypothesis we can now define a model for the alternate hypothesis for our sequential hypothesis test.

\section{Markov Model for Two Dependent vehicles}

Next we define the joint observation model under the hypothesis that vehicles are moving together as a convoy. This model makes the assumption that the transition times which $X$ and $Y$ make from their current states is independent from the vehicles which are traveling as a convoy. With this assumption, we can see that the waiting times (including the time either vehicle remains at its current state) will factor out leaving the 
likelihood as

$$
\begin{aligned}
p_{t}(X(t) & \left.Y(t) \mid H_{1}\right) \\
=p_{t} & \left(\left\{t_{k}^{x}\right\}_{k=1}^{n_{x}(k)} \mid\left\{x_{k}\right\}_{k=1}^{n_{x}(k)}\right) \times p_{t}\left(\left\{t_{k}^{y}\right\}_{k=1}^{n_{y}(k)} \mid\left\{y_{k}\right\}_{k=1}^{n_{y}(k)}\right) \\
& \times\left(1-F_{\tau}\left(t-t_{k}^{x} \mid x_{k}\right)\right) \times\left(1-F_{\tau}\left(t-t_{k}^{y} \mid y_{k}\right)\right) \\
& \times p_{t}\left(x_{k}, y_{k}, m_{k} \mid\left(x_{j}, y_{j}, m_{j}\right)_{j<k}, H_{1}\right),
\end{aligned}
$$

where we introduce a new variable $m_{i}$ which can take one of two values $\{X, Y\}$ and indicates either that vehicle $X$ or vehicle $Y$ transitioned at instance $i$. The probability $p_{t}\left(x_{k}, y_{k}, m_{k} \mid\left(x_{j}, y_{j}, m_{j}\right)_{j<k}, H_{1}\right)$ also satisfies the Markov property in that it can be expressed as

$$
\begin{aligned}
& p_{t}\left(x_{k}, y_{k}, m_{k} \mid\left(x_{j}, y_{j}, m_{j}\right)_{j<k}, H_{1}\right) \\
& \quad=p_{t}\left(x_{k}, y_{k}, m_{k} \mid x_{k-1}, y_{k-1}, m_{k-1}, H_{1}\right)
\end{aligned}
$$

where the current probability only depends on the previous observation. Now all that remains to define is this density $p_{t}\left(x_{k}, y_{k}, m_{k} \mid x_{k-1}, y_{k-1}, m_{k-1}, H_{1}\right)$ which we call the lag density.

1) Lag Density: The lag density,

$$
p_{t}\left(x_{k}, y_{k}, m_{k} \mid x_{k-1}, y_{k-1}, m_{k-1}, H_{1}\right),
$$

is only described under the alternate hypothesis. This term needs to be designed to capture the correlation between vehicles $X$ and $Y$ under $H_{1}$. This density is described by the changes in physical distances between the states in $S$ between observations of $X$ and $Y$ under $H_{1}$.

Let $\operatorname{dist}\left(x_{i}, y_{i}\right)$ denote the physical distance between states $x_{i}$ and $y_{i}$ at instant $i$. We assume the sensor positions are known.

Now one can look at how this distance property changes between observations of either $X$ or $Y$ with

$$
\delta_{i}=\frac{\operatorname{dist}\left(x_{i-1}, y_{i-1}\right)-\operatorname{dist}\left(x_{i}, y_{i}\right)}{\operatorname{dist}\left(x_{i-1}, y_{i-1}\right)} .
$$

The term $\delta_{i}$ describes the change in the lag property between observations $i-1$ and $i$ and is a signed value in the range $(-\infty, 1]$. We then introduce a new parameter $L$ which is the maximum allowable lag. Based on this parameter, this density will be able to capture when two vehicles are close together and one transitions away from the pair. Now if the previous distance $\operatorname{dist}\left(x_{i-1}, y_{i-1}\right)$ is less than some quantity $L$ then this means that at instance $i-1$, vehicles $X$ and $Y$ were close together, or at least within $L$ distance of each other. Then at instance $i$, either vehicle $X$ or $Y$ will transition away and the current distance will change. In this case, we consider that whoever transitions away when the previous distance was less than $L$ is assumed traveling independent of the pair. This introduces a notion of "leading" since whoever transitions away first will lead the other.

Now in the alternate case, when the previous distance is at least $L$ large, we look at the new distance to see if it has shrank or grown. If it has grown larger than $2 \times \operatorname{dist}\left(x_{i-1}, y_{i-1}\right)$ then the likelihood is forced to the null, i.e. the probability that the pair travels as a convoy goes to 0 , since that would mean that the other vehicle in the pair has moved further away from the "leader".

Otherwise the probability of a convoy is set proportional to the distance covered by the transition. This translates to

$$
\begin{aligned}
p_{t} & \left(x_{k}, y_{k} \mid x_{k-1}, y_{k-1}, m_{k-1}, m_{k}=X, H_{1}\right) \\
& =p_{t}\left(x_{k} \mid x_{k-1}, y_{k-1}, m_{k-1}, m_{k}=X, H_{1}\right) \\
& \propto \begin{cases}\operatorname{Pr}\left(x_{k} \mid x_{k-1}\right) & , \operatorname{dist}\left(x_{k-1}, y_{k-1}\right)<L \\
\frac{1+\delta_{i}}{2} & ,\left(-1 \leq \delta_{i}\right) \wedge\left(\operatorname{dist}\left(x_{k-1}, y_{k-1}\right) \geq L\right) \\
0 & ,(-1>\delta) \wedge\left(\operatorname{dist}\left(x_{k-1}, y_{k-1}\right) \geq L\right)\end{cases}
\end{aligned}
$$

if $m_{k}=X$ and

$$
\begin{aligned}
p_{t} & \left(x_{k}, y_{k} \mid x_{k-1}, y_{k-1}, m_{k-1}, m_{k}=Y, H_{1}\right) \\
& =p_{t}\left(y_{k} \mid x_{k-1}, y_{k-1}, m_{k-1}, m_{k}=Y, H_{1}\right) \\
& \propto \begin{cases}\operatorname{Pr}\left(y_{k} \mid y_{k-1}\right) & , \operatorname{dist}\left(x_{k-1}, y_{k-1}\right)<L \\
\frac{1+\delta_{i}}{2} & ,\left(-1 \leq \delta_{i}\right) \wedge\left(\operatorname{dist}\left(x_{k-1}, y_{k-1}\right) \geq L\right) \\
0 & ,(-1>\delta) \wedge\left(\operatorname{dist}\left(x_{k-1}, y_{k-1}\right) \geq L\right)\end{cases}
\end{aligned}
$$

if $m_{k}=Y$. The first of the two cases in these expressions, when $\operatorname{dist}\left(x_{i-1}, y_{i-1}\right)<L$, is already a valid discrete density since it is just the likelihood of the previous transition, however the alternate piece will need to be normalized so that it sums to one. This density is discrete because it can take one of $|S|^{3}$ values since it can only be a transition of $X$ or $Y$ given $X$ and $Y$ 's previous states. This linear density transformation states that if the previous lag was large, then the smaller the new lag is the more likely the pair is a convoy.

\section{E. Formulation of a Likelihood Ratio}

Now that both probabilities are defined under the null and alternate hypothesis, one can formulate a hypothesis test between the two. This is formulated with the following likelihood ratio

$$
\begin{aligned}
\Lambda & \left(Z\left(t_{i}\right) \mid Z\left(t_{i-1}\right)\right) \\
& =\frac{\operatorname{Pr}\left(Z\left(t_{i}\right)=z_{i} \mid Z\left(t_{i-1}\right)=z_{i-1}, H_{1}\right)}{\operatorname{Pr}\left(Z\left(t_{i}\right)=z_{i} \mid Z\left(t_{i-1}\right)=z_{i-1}, H_{0}\right)} .
\end{aligned}
$$

Transforming Equation 10 into the log-domain and expanding the inner probabilities this simplifies to

$$
\begin{aligned}
\ln \Lambda & \left(Z\left(t_{i}\right) \mid Z\left(t_{i-1}\right)\right) \\
= & \ln \operatorname{Pr}\left(x_{n_{x}\left(t_{i}\right)}, y_{n_{y}\left(t_{i}\right)} \mid x_{n_{x}\left(t_{i-1}\right)}, y_{n_{y}\left(t_{i-1}\right)}\right) \\
& \quad-\ln \operatorname{Pr}\left(x_{n_{x}\left(t_{i}\right)} \mid x_{n_{x}\left(t_{i-1}\right)}\right)-\ln \operatorname{Pr}\left(y_{n_{y}\left(t_{i}\right)} \mid y_{n_{y}\left(t_{i-1}\right)}\right)
\end{aligned}
$$

where it can be noted that the waiting times have canceled out due to the model definition. Now the likelihood ratio definition only depends on the actual transitions and not the time of the transition. Given a vector of $\mathbf{Z}_{n}$ observations the ratio becomes

$$
\begin{aligned}
& \ln \Lambda\left(\mathbf{Z}_{n}\right)=\ln \pi\left(z_{0} \mid H_{1}\right)-\ln \pi\left(z_{0} \mid H_{0}\right) \\
& \quad+\sum_{i=1}^{n} \ln \operatorname{Pr}\left(x_{n_{x}\left(t_{i}\right)}, y_{n_{y}\left(t_{i}\right)} \mid x_{n_{x}\left(t_{i-1}\right)}, y_{n_{y}\left(t_{i-1}\right)}\right) \\
& \quad-\sum_{i=1}^{n}\left[\ln \operatorname{Pr}\left(x_{n_{x}\left(t_{i}\right)} \mid x_{n_{x}\left(t_{i-1}\right)}\right)+\ln \operatorname{Pr}\left(y_{n_{y}\left(t_{i}\right)} \mid y_{n_{y}\left(t_{i-1}\right)}\right)\right]
\end{aligned}
$$


where $\pi\left(z_{0} \mid H_{h}\right)$ is the initial probability of $z_{0}$ under the hypothesis $H_{h}, h \in 0,1$. In this model it is assumed that the initial probabilities under the null and alternate hypothesis are both equal and therefore cancel out as well.

1) Recursive Definition of the Likelihood Ratio: In order for this model to make decisions as quickly as possible, we would like to translate the standard hypothesis test of

$$
\Lambda(X(t), Y(t))=\frac{p_{t}\left(X(t), Y(t) \mid H_{1}\right)}{p_{t}\left(X(t), Y(t) \mid H_{0}\right)}
$$

into a sequential hypothesis test which receives data as it becomes available. In order to define a sequential hypothesis test, there needs to be a definition of the likelihood ratio without all the data available. The likelihood ratio can be defined recursively using the previous sample of the likelihood ratio as

$$
\begin{aligned}
& \ln \Lambda\left(x_{k}, y_{k}\right)=\ln \Lambda\left(x_{k-1}, y_{k-1}\right) \\
& \quad+\ln p_{t}\left(x_{k}, y_{k}, m_{k} \mid x_{k-1}, y_{k-1}, m_{k-1}, H_{1}\right) \\
& \quad-\ln p_{t}\left(x_{k} \mid x_{k-1}, H_{0}\right)-\ln \operatorname{Pr}\left(y_{k} \mid y_{k-1}, H_{0}\right)
\end{aligned}
$$

where the likelihood ratio at time $t_{k}$ only depends on the current observation of $x_{k}$ and $y_{k}$ and the previous observation and likelihood ratio at time $t_{k-1}$. One can further simplify this into two separate cases depending on the observation. The cases appear as

$$
\begin{aligned}
& \ln \Lambda\left(x_{k}, y_{k}\right)=\ln \Lambda\left(x_{k-1}, y_{k-1}\right)+ \\
& \left\{\begin{aligned}
\ln \operatorname{Pr}\left(x_{k} \mid x_{k-1}, y_{k-1}, H_{1}\right) & \\
-\ln \operatorname{Pr}\left(x_{k} \mid x_{k-1}, H_{0}\right) & , \\
\ln \operatorname{Pr}\left(y_{k} \mid x_{k-1}, y_{k-1}, H_{1}\right) & \\
-\ln \operatorname{Pr}\left(y_{k} \mid y_{k-1}, H_{0}\right) & , \quad m_{k}=Y .
\end{aligned}\right.
\end{aligned}
$$

This simplification shows that since only one vehicle (either $X$ or $Y$ ) can transition at any time, the update rules for the likelihood ratios are easy and fast to compute with only the previous likelihood ratio being necessary in order to compute the current likelihood ratio. It also shows how the lag property is related to individual path probabilities for observations of each random vehicle.

\section{F. Sequential Hypothesis Test}

Now that a recursive definition of the log likelihood ratio is available from Equation 11 a sequential hypothesis test can be defined. As per the standard literature on sequential hypothesis testing [2] two boundaries need to be defined, $\ln \eta_{0}$ and $\ln \eta_{1}$, which define the decision regions for the test. The following equation defines how the boundaries define the decision regions.

$$
\begin{aligned}
\ln \Lambda\left(x_{k}, y_{k}\right)<\ln \eta_{0} & \text { decide } H_{0} \\
\ln \eta_{0} \leq & \ln \Lambda\left(x_{k}, y_{k}\right)<\ln \eta_{1} \\
\ln \eta_{1} \leq \ln \Lambda\left(x_{k}, y_{k}\right) & \text { decide "need more data" } H_{1}
\end{aligned}
$$

\section{Simulations AND Results}

A simulated dataset where real traffic patterns are modelled was created to test the performance of this algorithm. In this dataset the number of convoys was controllable as well as the decision bounds in order to determine the performance of the algorithm accurately. With simulated data the false detection and detection probabilities are able to be analysed along with the computational performance.

\section{A. Dataset Creation}

The simulated dataset was created by selecting 48 sensors at random locations in the borough of LaSalle in Montreal, Quebec, Canada. These locations constitute the state locations for the underlying sensor network which was created. When simulated vehicles pass by these locations we can simulate a detection of that vehicle and record it.

Then using the Visum [6] simulator, which simulates traffic load across all roads in Montreal, a random population of vehicles was created equal to the number of registered cars in Montreal. The cars selected were sent traveling through the network for 1 day and when a vehicle was reported to have passed a simulated sensor location it was recorded into the dataset.

This created a sample dataset of base data where no convoys were known. From this a set of simulated convoys were injected into this dataset following the proposed alternate hypothesis joint state transition model. There are 1000 convoy pairs which were simulated and injected. In each pair, times between observations were randomly generated from a uniform distribution between -60 seconds and 60 seconds. If this random observation time is negative, it means that vehicle $Y$ was observed prior to vehicle $X$ and therefore $X$ is following $Y$. Each pair was simulated for 18 samples of the convoy.

\section{B. Algorithm Analysis on Simulated Data}

Utilizing the simulated dataset with 1000 convoys injected as described, the algorithm was run with the decision boundaries being swept for values $0 \leq \ln \eta_{1} \leq 22$ and $0 \geq \ln \eta_{0} \geq$ -16 . The number of detections of pairs of vehicles from $H_{1}$, false detections (pairs of vehicles reported to be from $H_{1}$ but truly from $H_{0}$ ), as well as non-decisions (pairs of vehicles likelihood ratios whose value never moved into either decision boundary) were recorded. From this information, Figure 2 relates how the percent of detection for convoys changes with the changes in $\ln \eta_{0}$ and $\ln \eta_{1}$.

Similarly Figure 3 demonstrates how for all combinations of $\ln \eta_{0}$ and $\ln \eta_{1}$ the resulting combination of $P_{D}$ and $P_{F D}$ is produced. Taking the largest output for $P_{D}$, which occurs at $\ln \eta_{0}=-17.5$ and $\ln \eta_{1}=0$, one can see that there is still only $P_{F D}=0.0383$ which is quite low in order to attain a $P_{D}=$ 0.8550 . This proves to be one of the most useful comparisons when choosing decision boundaries for the system. In order to minimize the number of inaccurate decisions of convoys and maximizing the number of detected real convoys, this chart helpfully displays the trade-off and can allow an operator to choose $\ln \eta_{0}$ and $\ln \eta_{1}$ for the target performance they wish to achieve.

One can also view the amount of data which is not a decision for $H_{0}$ nor $H_{1}$. This means that the tracked pair of vehicles 


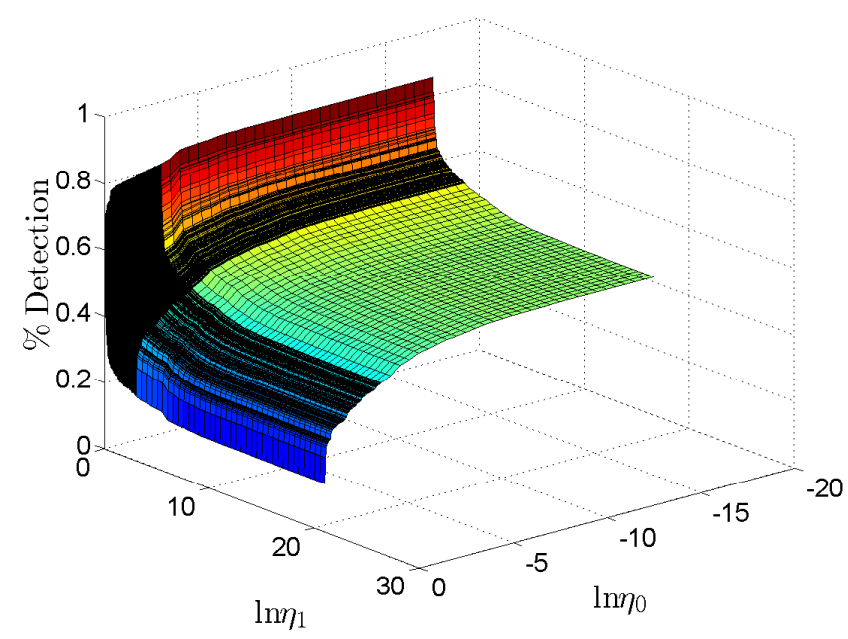

Fig. 2. ROC surface plot demonstrating how the choice of $\ln \eta_{0}$ and $\ln \eta_{1}$ changes the detection accuracy of the system.

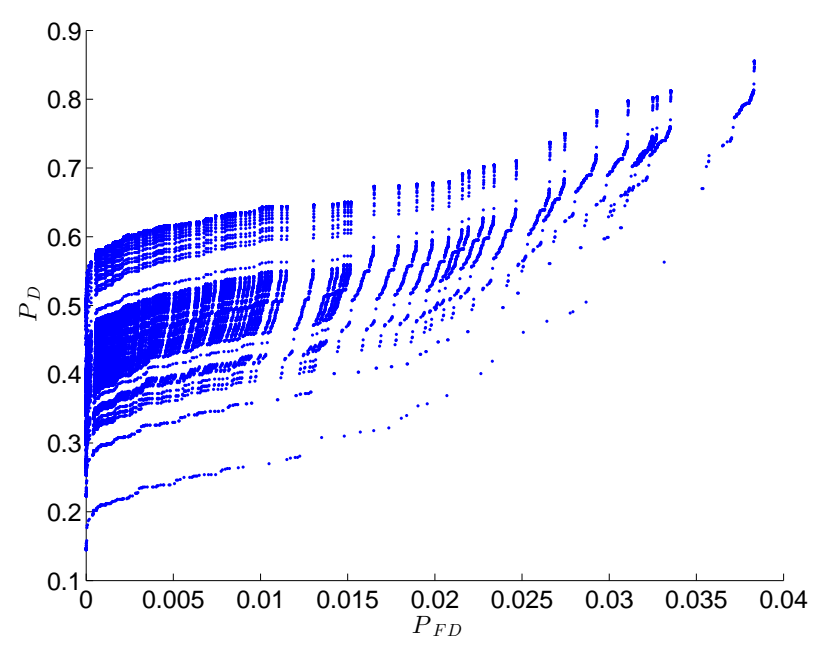

Fig. 3. Plot of the values showing the probability of detection $P_{D}$ versus the probability of false detection $P_{F D}$ for this system in the simulated dataset.

was lost when there were no more samples before the track time window expired.

Figure 4 demonstrates this parameter through changing values of $\ln \eta_{0}$ and $\ln \eta_{1}$. As one pushes the boundary parameters further from $0\left(\ln \eta_{0} \rightarrow-\infty\right.$ and $\left.\ln \eta_{1} \rightarrow \infty\right)$ one can see that the percent of data which is no-decision tends towards $100 \%$. This makes sense where data is not infinite since any single pair will only have a finite log likelihood.

Lastly Figure 5 shows a heat map which demonstrates how the average number of observations until a decision of vehicles changes with the boundaries $\ln \eta_{0}$ and $\ln \eta_{1}$. The average number of observations is how many samples of the pair of vehicles must be observed before a decision for the alternate case $H_{1}$ can be declared. As can be seen in the heat map as the boundaries move farther from 0 the number of observations required to make a decision increases.

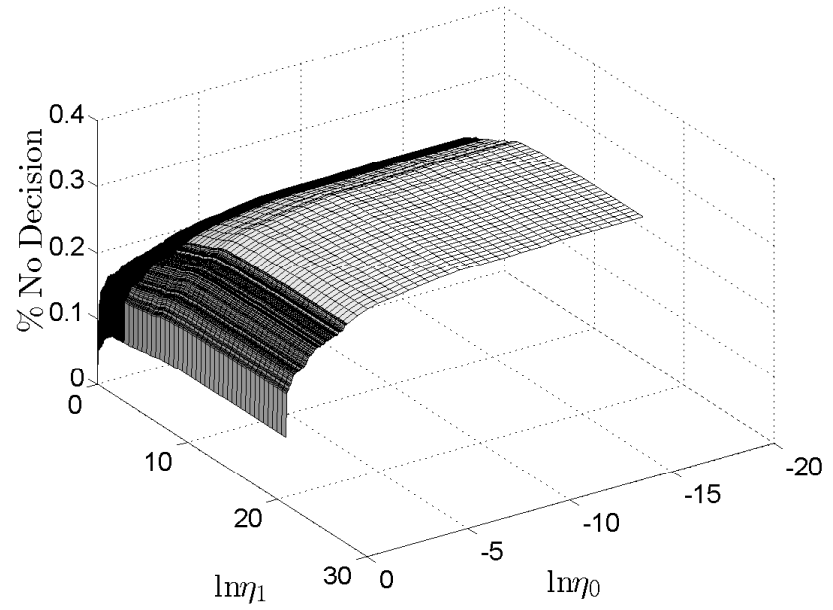

Fig. 4. ROC surface plot demonstrating how the choice of $\ln \eta_{0}$ and $\ln \eta_{1}$ changes the percent of non-detections in the system.

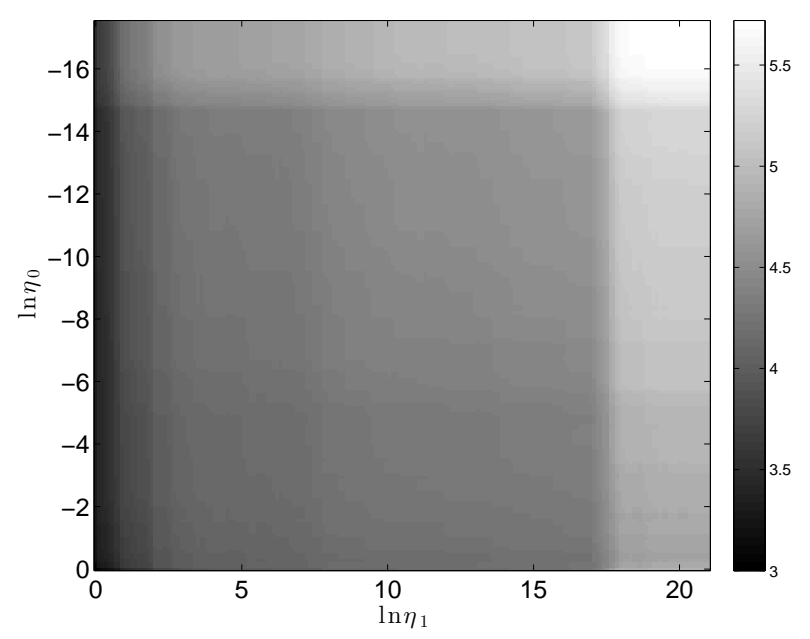

Fig. 5. Heatmap demonstrating how the average number of decisions required to make a decision for "convoy" changes with boundaries $\ln \eta_{0}$ and $\ln \eta_{1}$.

\section{COnClusions And Future Work}

The algorithm presented here utilizes standard sequential hypothesis testing techniques [2] in order to create a method for detecting pairs of vehicles traveling in a convoy versus traveling independently through a network of nodes. The main contribution for this work is the definition of a model which describes how vehicles move co-dependently with each other through a network of states of a Markov chain. Analysis over a real dataset is demonstrated showing that a real convoy was detected. However no performance analysis could be done since the ground truth is unknown in this dataset. Therefore a simulated dataset where the exact convoy pairs are known was created and the algorithm was evaluated on that dataset. The results of this analysis were presented showing that the algorithm has the ability to detect convoys with varying success depending on the acceptable percentage of false detections for 
the specific application.

In the future, the model will be extended in order to take into account the transition times of vehicles. Since the current model assumes that all transition times are independent of the underlying distribution $H_{1}$ or $H_{0}$. Extending this model to include the transition times would hopefully make it more accurate and help push the performance closer to the optimal $P_{D}=1$ and $P_{F D}=0$.

\section{REFERENCES}

[1] S. Lawlor, "Detecting cconvoy in networks of short-range sensors," Master's thesis, McGill University, 2013.

[2] A. Wald, Sequential Analysis, ser. Wiley Publication In Statistics, R. A. Bradley, J. S. Hunter, D. G. Kendall, and G. S. Watson, Eds. John Wiley \& Sons, Inc., 1966.

[3] E. Pollard, B. Pannetier, and M. Rombaut, "Convoy detection processing by using the hybrid algorithm (gmcphd/vs-immc-mht) and dynamic bayesian networks," in International Conference on Information Fusion, vol. 12, Seattle, WA, USA, July 2009.

[4] W. Koch, "Information fusion aspects related to GTMI convoy tracking," in Information Fusion, 2002. Proceedings of the Fifth International Conference on, vol. 2, 2002, pp. 1038-1045.

[5] R. W. Sinnott, "Virtues of the haversine," Sky and Telescope, vol. 68, p. 158, 1984.

[6] T. Sider, A. Alam, M. Zukari, H. Dugum, N. Goldstein, N. Eluru, and M. Hatzopoulou, "Land-use and socio-economics as determinants of traffic emissions and individual exposure to air pollution," Journal of Transport Geography, vol. 33, pp. 230 - 239, 2013. 